\section{MS13-P3 Crystal Structure of the cytoplasmic portion of histidine kinase SrrB from Staphylococcus aureus Wen-Yih Jeng ${ }^{1,2}$, Ya-Jin Jheng ${ }^{1,2}$, Chia-I Liu ${ }^{3}$, Chieh-Shan Lee ${ }^{1,2}$,
Te-Jung Lu ${ }^{4}$}

1. University Center for Bioscience and Biotechnology, National Cheng Kung University, Tainan 701, Taiwan

2. Department of Biochemistry and Molecular Biology, National Cheng Kung University, Tainan 701, Taiwan

3. School of Medical Laboratory Science and Biotechnology, Taipei Medical University, Taipei 110, Taiwan

4. Department of Medical Laboratory Science and Biotechnology, Chung Hwa University of Medical Technology, Tainan 717, Taiwan

email: wyjeng@mail.ncku.edu.tw

Staphylococcus aureus is one of the major causes of nosocomial infections today. $S$. aureus infection is considered to be the reason for the increasing number of antibiotic-resistant strains. Furthermore, S. aureus can produce slime layer or multilayered biofilm embedded with the glycocalyx on the surface of various biomaterials. $S$. aureus can persist in clinical settings and increase resistance to antimicrobial agents through biofilm formation. The staphylococcal respiratory response regulator $\operatorname{SrrAB}^{-}$(synonym, $\overline{\mathrm{SrhSR}}$ ), a two-component system has been identified as a global regulator of the aerobic-anaerobic shift of metabolism in $S$. aureus. SrrB is a histidine kinase which responds to environmental stimuli by regulating its cognate response regulator SrrA. Under low-oxygen growth conditions, the staphylococcal regulator SrrAB induces ica locus transcription and polysaccharide intercellular adhesion (PIA) production, known as a major component of biofilm. SrrB is a transmembrane sensor protein consisted of 588 residues $(66.7 \mathrm{kDa})$. N-terminal hydrophilic region of SrrB is located extracellularly, while the C-terminal phosphoacceptor, dimerization, and ATPase domains are located intracellularly. Here, we report the crystal structure of cytoplasmic portion of $\mathrm{SrrB}(\mathrm{SrrBc})$ from $S$. aureus in a dimeric form at $1.78 \AA$ resolution. The overall structure of $\mathrm{SrrBc}$ forms a unique homodimer which is mediated by two pairs of long dimerization $\alpha$-helices. To the rear of dimerization helices is the ATP binding domain of SrrB which consists of three parallel $\alpha$-helices, one short anti-parallel $\beta$-sheet, one core mixed type $\beta$-sheet and a disordered ATP-lid region. Moreover, one disulfide bond (Cys469 and Cys506) is located within the core $\beta$-sheet to stable the structure. Finally, based on previous studies and our crystal structure, we propose that the redox state of this disulfide bond in the ATP binding domain of SrrB might play a key role to mediate the interaction between SrrB and SrrA, thus alter the target gene of SrrAB in transcription level in S. aureus.

Keywords: biofilm, multiple antibiotic resistant, two-component system, MRSA, VISA, PIA
MS13-P4 Structural studies of AggC, an novel O-GlcNAc transferase involved in protection of virulence-associated cell proteins in Staphylococcus aureus Chia-I Liu ${ }^{1}$, Wen-Yih Jeng ${ }^{2}$, Tzu-Ping Ko ${ }^{3}$, Andrew H.-J. Wang ${ }^{3,4}$

1. School of Medical Laboratory Science and Biotechnology, College of Medical Science and Technology, Taipei Medical University, Taipei 10031, Taiwan

2. University Center for Bioscience and Biotechnology, National Cheng Kung University, Tainan 70101, Taiwan

3. Institute of Biological Chemistry, Academia Sinica, Taipei 11529, Taiwan

4. Graduate Institute of Translational Medicine, College of Medical Science and Technology, Taipei Medical University, Taipei 10031, Taiwan

email: ponpiqq@gmail.com

Glycosylation of bacterial cell wall proteins play a critical role in bacterial pathophysiology. Two novel O-linked glycosyltransferases (OGTs), AggB and AggC, decorate all SD (serine-aspartate) repeats of adhesins with $\mathrm{N}$-acetylglucosamine (GlcNAc) moieties, which containing the virulence factors ClfA (a fibrinogen-binding clumping factor A), ClfB, SdrC (SD repeats C), SdrD, SdrE of $S$. aureus and $\mathrm{SdrF}, \mathrm{SdrG}$, SdrH of $S$. epidermidis. Recently, it has been demonstrated that glycosylated SD repeats proteins can facilitate bacterial adhesion, immune evasion, colonization, persistence and invasion of host tissue. This specific modification also promotes $S$. aureus replication in the bloodstream of mammalian hosts. AggB and AggC modify all SD repeats proteins by an ordered mechanism, with AggC appending the sugar residues proximal to the target SD repeats, followed by additional modification by AggB. Here we report two crystal structures of $S$. aureus AggC, as a binary complex with citrate $(2.8 \AA)$ and as a ternary complex with UDP and GlcNAc (2.2 ̊). The structures provide clues to the enzyme catalytic mechanism, implying how AggC recognizes target peptide sequences, and reveal the fold of the unique $\beta$-meander domain and a core catalytic domain with GT-B fold. Structure-based mutagenesis of AggC was also performed to explore the roles of amino acids involved in substrate binding. In summary, this information will accelerate the rational design of biological experiments to investigate AggC functions and also help the design of inhibitors as a therapeutic target.

Keywords: serine-aspartate (SD) repeats, glycosyltransferase, virulence factor, X-ray Crystallography 